\title{
PP2A enables IL-2 signaling by preserving IL-2R $\beta$ chain expression during Treg development
}

\author{
Amir Sharabi, ${ }^{1,2,3}$ Hao Li, ${ }^{1}$ Isaac R. Kasper, ${ }^{1}$ Wenliang Pan, ${ }^{1}$ Esra Meidan, ${ }^{1}$ Maria G. Tsokos, ${ }^{1}$ \\ Vaishali R. Moulton, ${ }^{1}$ and George C. Tsokos ${ }^{1}$ \\ 'Division of Rheumatology and Clinical Immunology, Department of Medicine, Beth Israel Deaconess Medical Center, \\ Harvard Medical School, Boston, Massachusetts, USA. ${ }^{2}$ Department of Rheumatology, Campus Beilinson, The Rabin \\ Medical Center, Petach Tikva, Israel. ${ }^{3}$ Department of Microbiology and Immunology, Sackler Faculty of Medicine, Tel Aviv \\ University, Tel Aviv, Israel.
}

Tregs require IL-2 signaling for signal transducer and activator of transcription 5-mediated (STAT5mediated) induction of Foxp3. Although phosphatase 2A (PP2A) is a negative regulator of IL-2 production in effector T cells and Tregs do not produce IL-2, it is not known whether PP2A controls IL-2 signaling in Tregs. To explore the role of PP2A in IL-2 signaling in Tregs, we studied mice engineered to lack PP2A in all Foxp3-expressing cells. We report that PP2A is required to enable Foxp3 expression and to maintain sufficient numbers of Tregs in the thymus. We show for the first time to our knowledge that PP2A prevents the selective loss of surface IL-2R $\beta$ and preserves IL-2R signaling potency in Tregs. The loss of IL-2R $\beta$ in thymus- and spleen-derived Tregs that lack PP2A is because of increased sheddase activity. Pan-sheddase or selective ADAM10 (a disintegrin and metalloproteinase 10) inhibition, like forced expression of IL-2R $\beta$ in PP2A-deficient Tregs, restored IL-2R $\beta$ expression and signaling. Thus, PP2A restrains the sheddase activity of ADAM10 in Tregs to prevent the cleavage of IL-2Rß from the cell surface to enable competent IL-2R signaling, which is essential for Tregs' development and homeostasis.

Conflict of interest: The authors have declared that no conflict of interest exists.

Copyright: (c) 2019 American Society for Clinical Investigation

Submitted: November 19, 2018 Accepted: March 19, 2019 Published: May 2, 2019.

Reference information: /Cl Insight. 2019;4(9):e126294. https://doi. org/10.1172/ji.insight.126294.

\section{Introduction}

IL-2 signaling is indispensable for the induction of FOXP3 and the differentiation of Tregs in the thymus (1-6). Loss of IL-2 receptor (IL-2R) signaling $(3,7)$ results in profound Treg impairment and autoimmunity. The high-affinity IL-2R is composed of 3 subunits: the $\alpha$ chain (IL-2R $\alpha$; CD25), which is highly expressed by Tregs; the $\beta$ chain (IL-2R $\beta$; CD122); and the $\gamma$ chain (IL-2R $\gamma$; CD132), all of which are present on the surface membrane of Tregs without forming stable heterotrimers. IL-2 is first captured by IL-2R $\alpha$ with a relatively weak affinity $\left(K_{D} 10^{-8} \mathrm{M}\right)$, which then associates with IL-2R $\beta$ and IL-2R $\gamma$ chains to produce a stable quaternary high-affinity IL-2R $\left(K_{D} 10^{-11} \mathrm{M}\right)(8-12)$.

Tregs are unable to produce IL-2; however, they selectively exhibit high affinity for IL-2 through mechanisms that are not well understood. It has been suggested that the higher affinity of Tregs for IL-2 stems from the expression of higher levels of IL-2R $\alpha$ and IL-2R $\gamma$ chains and protein phosphatase activity, including protein phosphatase 2A (PP2A) (13). PP2A is a highly conserved serine/threonine phosphatase that regulates diverse pivotal cellular processes $(14,15)$. Although PP2A is a negative regulator of IL-2 production in T effector cells (16), it is required for proper Treg function (17). Nevertheless, the role of PP2A in IL-2 signaling in Tregs is unknown. A competent machinery for IL-2 signaling is critical for Tregs because tyrosine phosphorylation of the downstream signaling partner of STAT5 is indispensable for inducing FOXP3 expression (1-6). To address the role of PP2A in IL-2 signaling and Tregs' development, we studied mice engineered to lack PP2A in all Foxp3-expressing cells. Here we report that PP2A enables IL-2 signaling in Tregs by restraining the sheddase activity of ADAM10 (a disintegrin and metalloproteinase 10) and preventing the cleavage of IL-2R $\beta$ from the cell surface.

\section{Results}

$C D 4^{+}$Tregs require PP2A to maintain their prevalence in the thymus. Because Foxp $3^{y p . C r e} P p p 2 r 1 a^{\mathrm{f} / \mathrm{fl}}$ mice (KO 
mice) develop systemic autoimmunity (including lymphadenopathy; inflammatory cell infiltration into the skin, intestine, pancreas, liver, and lungs; and the production of autoantibodies) at the age of 10 to 14 weeks while Foxp $3^{\text {Cre }} P p p 2 r 1 a^{\mathrm{wt} / \mathrm{wt}}$ mice (WT mice) remain healthy (17), we used Foxp3yp.Cre reporter mice to ask whether PP2A contributes to $\mathrm{CD}^{+}$Treg development in the thymus. The vast majority of FOXP3 expression in the thymus appears at the CD4-single-positive (CD4-SP) cell compartment (18); therefore, the frequency of $\mathrm{CD}^{+}$Tregs was determined in the Thy $1.2^{+} \mathrm{CD}^{+} \mathrm{CD} 4^{+} \mathrm{CD} 8^{-}-\mathrm{SP}$ FOXP3-yellow fluorescent protein ${ }^{+}\left(\mathrm{YFP}^{+}\right)$cell compartment. Flow cytometry plots and a graphic summary of individual values ( $n=4-6$ mice/group) for $\mathrm{CD}^{+}$Treg percentages from a representative of 3 experiments are shown in Figure $1 \mathrm{~A}$. KO mice with established disease had a significant $50 \%$ decrease in the mean percentages of thymic $\mathrm{CD}^{+}$Tregs in comparison with WT mice (from $2.7 \% \pm 0.4 \%$ to $1.2 \% \pm 0.1 \%, P<0.005$ ). In addition, thymic PP2A-deficient CD4 ${ }^{+}$Tregs displayed significantly decreased (by $85 \%, P<0.005$ ) levels of Foxp3 mRNA (Figure 1B) and protein levels (Figure 1C and Supplemental Figure 1; supplemental material available online with this article; https://doi.org/10.1172/jci.insight.126294DS1).

$I L-2-$ mediated induction of FOXP3 in thymic CD4 $4^{+}$Tregs involves PP2A. The generation of thymic CD25 cells is a T cell receptor-instructive (TCR-instructive) event $(19,20)$, and thymic CD4 ${ }^{+}$Tregs develop in a 2-step process wherein TCR signals result in the expression of IL-2R $\alpha$ chain (CD25) and the induction of Foxp3 (21). Therefore, we determined the expression of CD25 in thymic CD4 ${ }^{+}$Tregs from the KO and WT mice. Indeed, thymic CD4 ${ }^{+}$Tregs from KO mice expressed CD25 at higher levels $(P<0.01)$ compared with WT mice (Figure 1D), a finding that eliminates a defect in TCR signaling.

CD25 $5^{\text {hi }}$-expressing cells in the thymus precede those expressing Foxp3 (22), and this transition requires IL-2 signaling (21). Therefore, thymic precursors of CD4+ Tregs defined as CD4-SP, CD25 $5^{\text {hi }}$, FOXP3-YFP- cells were sorted from KO and WT mice and challenged with or without IL-2 in plates precoated with $\alpha$-CD3 for 18 to 24 hours. Although Foxp3 expression, as determined by immunoblotting of cell lysates (described in Methods), was induced in cells from both WT and KO mice, it lagged significantly $(P<0.02)$ in PP2A-deficient cells (Figure $1 \mathrm{E}$ ). Taken together, these data suggest that deficiency of PP2A in thymic CD4+ Tregs significantly decreases their frequency in the thymus and interferes with the ability of their precursors to acquire Foxp3.

IL-2 signaling in thymic CD4 ${ }^{+}$Tregs is PP2A dependent. IL-2 and the downstream transcription factor STAT5 are indispensable for the induction of Foxp3 and the differentiation of CD4 ${ }^{+}$Tregs in the thymus $(1-4)$. Phosphorylated STAT5 (p-STAT5) levels induced by IL-2 were found to be significantly $(P<0.001)$ reduced in thymic $\mathrm{CD}^{+}$Tregs from KO mice compared with WT (Figure 2A), suggesting that PP2A is required at this step. To exclude the possibility that thymic $\mathrm{CD} 4^{+}$Tregs from $\mathrm{KO}$ mice respond better to higher concentrations of IL-2, we performed a dose-response curve experiment (Figure 2B), which confirmed that PP2A-deficient Tregs respond poorly to IL-2, even at higher concentrations.

$P P 2 A$ controls the expression of the IL-2R $\beta$ chain in thymic $C D 4^{+}$Tregs. The IL-2R $\beta$ chain is mandatory for IL-2R signal transduction, and unlike in antigen-activated $\mathrm{T}$ cells it is constitutively expressed in $\mathrm{CD} 4^{+}$Tregs. Accordingly, we asked whether its expression is altered in Tregs deficient in PP2A. As shown in Figure 2C, $\mathrm{CD}^{+}$Tregs express significantly $(P<0.001)$ less IL-2R $\beta$ on the surface membrane compared with PP2A-sufficient $\mathrm{CD}^{+}$Tregs while the expression levels of the IL-2R $\gamma$ chain were comparable. The presence of the PP2A inhibitor okadaic acid in cultures of $\mathrm{CD}^{+}$Tregs from WT mice resulted in a significant $(P<0.02)$ reduction of IL-2R $\beta$ expression (Figure $2 \mathrm{D}$ ), confirming PP2A's importance in the expression of IL-2R $\beta$ on the surface membrane of Tregs. IL-2R $\beta$ phosphorylation requires the presence of the IL-2R $\gamma$ chain $(23,24)$. The fact that the total levels of phosphorylated IL-2R $\beta$ were decreased (Figure 2E and Supplemental Figure 1) in Tregs from KO mice while the ratio of phosphorylated versus nonphosphorylated IL-2R remained intact confirms that PP2A deficiency affects the expression of the IL-2R $\beta$ chain and not that of the IL-2R $\gamma$ chain.

$P P 2 A$ prevents the formation of soluble $I L-2 R \beta$ chain by thymic $C D 4^{+}$Tregs. Diminished protein levels of the IL-2R $\beta$ chain in thymic CD4 ${ }^{+}$Tregs from the KO mice could be the result of decreased production, increased internalization, or loss from the cell surface. The first possibility was excluded because the levels of Il2rb mRNA expression were increased in the cells from $\mathrm{KO}$ mice compared with those from WT mice (Figure 3A). Similarly, increased internalization of the IL-2R $\beta$ chain, which occurs upon binding of IL-2, could not account for its decreased expression on the surface membrane of PP2A-deficient Tregs. As shown in Figure 3B, although the IL-2R $\beta$ chain is quickly internalized in Tregs from WT mice, the low levels of the IL-2R $\beta$ chain in Tregs from KO mice remain stable after exposure to IL-2.

To study whether the IL-2R $\beta$ chain was shed from the cell surface, thymic CD4 ${ }^{+}$Tregs from the KO and the WT mice were cultured overnight in complete medium alone or in $\alpha$-CD3-precoated plates in the 
A
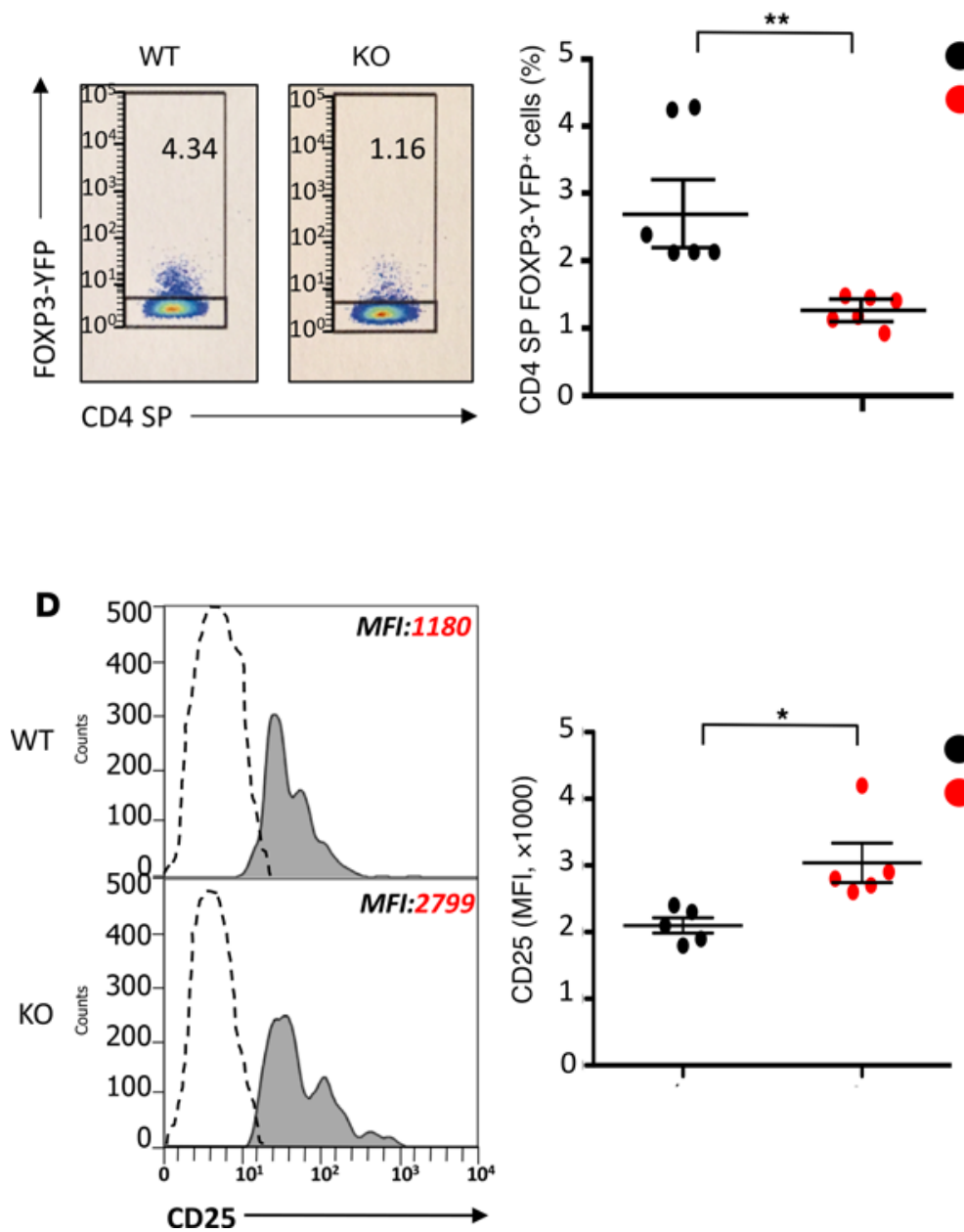

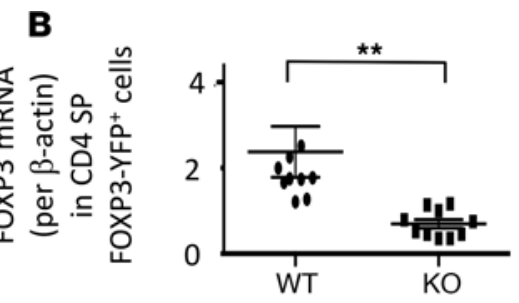

C

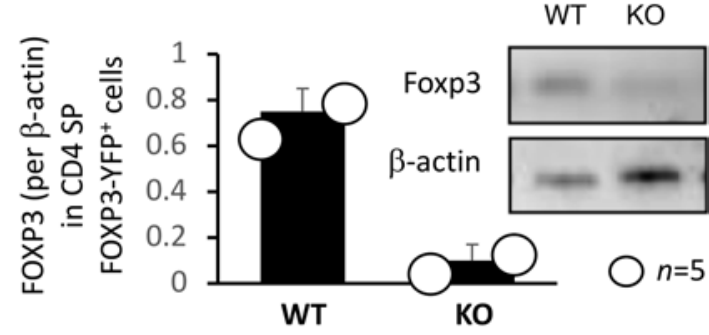

E
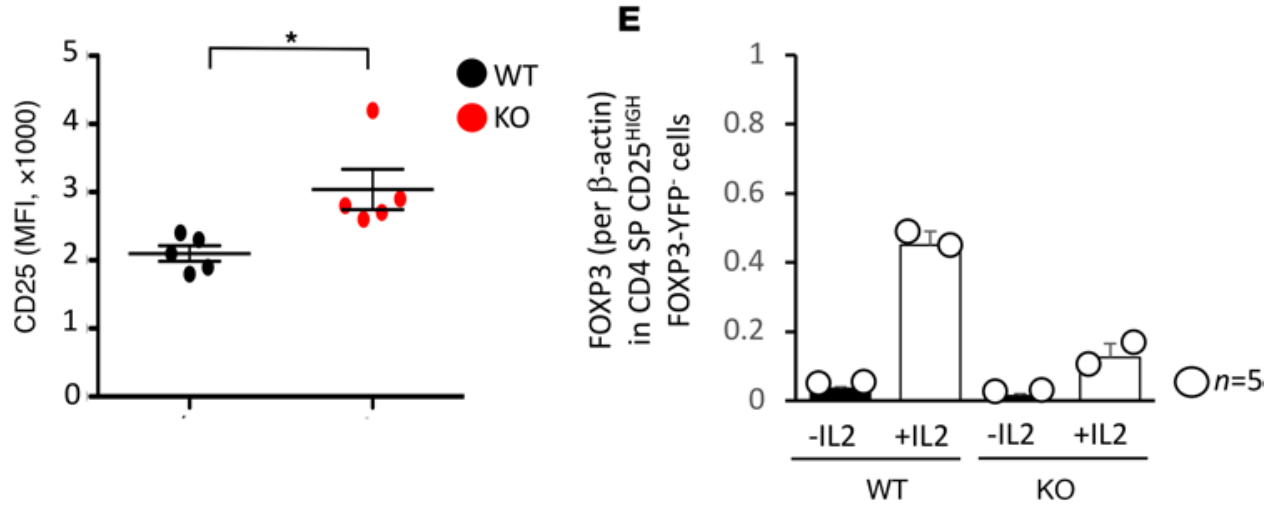

Figure 1. CD4 ${ }^{+}$Tregs require PP2A for thymic maintenance and IL-2-mediated induction of Foxp3. (A) Flow cytometry plots and cumulative data (mean \pm SEM) from individual mice ( $n=4-6 /$ group) depicting the percentages of thymic Thy1.2+CD3 ${ }^{+}$CD4 ${ }^{+}$CD8 $--S P$ FOXP3-YFP ${ }^{+}$CD4 ${ }^{+}$Tregs that are PP2A deficient (KO) or PP2A sufficient (WT). A representative of 3 experiments is shown. (B and C) Thymic CD4+ Tregs (defined as Th1.2+CD3+CD4+CD8--SP Foxp3-YFP+ cells) were sorted from individual KO and WT mice ( $n=5$ /group). (B) Total RNA from thymic CD4+ Tregs from individual KO and WT mice ( $n=4-5 / g r o u p)$ from 2 experiments was assessed for mRNA transcripts of Foxp3. Individual and cumulative data (mean \pm SEM) are shown. (C) Protein lysates were prepared following the pooling of cells in each group. The expression of Foxp3 and $\beta$-actin was assessed by Western blot analysis (right), and the Foxp3/ $\beta$-actin ratio (mean \pm SEM) was calculated from 2 independent experiments (left). (D) Expression of CD25 in thymic CD4+ Tregs from the KO and WT mice. Representative histograms (left) and cumulative data (right) from a representative of 3 experiments are shown (mean \pm SEM). Dashed-line histograms represent negative control. (E) Thymic precursors of CD4 ${ }^{+}$Tregs defined as Thy1.2+CD4+CD8-CD25 ${ }^{\text {hiFOXP3-YFP- }}$ cells were sorted from KO and WT mice (n $=5 /$ group $)$ and challenged with or without IL-2 $(20 \mathrm{mM})$ in plates precoated with $\alpha$-CD3 for 18 to 24 hours. Thereafter, the cells were pooled in each group and protein lysates were prepared. The expression of Foxp3 and $\beta$-actin was assessed by Western blot analysis (top), and the Foxp3/ $\beta$-actin ratio (mean \pm SEM) was calculated from 2 independent experiments (bottom). ${ }^{*} P<0.05 ;{ }^{* *} P<0.005$.

presence of IL-2, and thereafter the levels of soluble IL-2R $\beta$ (sIL-2R $\beta$ ) were determined in the supernatants. We found increased $(P<0.001)$ levels of sIL-2R $\beta$ in the culture supernatants of unstimulated thymic $\mathrm{CD}^{+}$Tregs from KO mice compared with those from WT mice, which increased further in the presence of IL-2 and $\alpha$-CD3 (Figure 3C). In agreement with this finding (Figure 3D), Tregs from WT mice cultured with the PP2A inhibitor okadaic acid increased the levels of sIL-2R $\beta$ significantly $(P<0.03)$, whereas in the presence of the PP2A activator FTY720, the levels of sIL-2R $\beta$ decreased significantly $(P<0.04)$. The functionality of both okadaic acid and FTY720 were confirmed in prior experiments (Supplemental Figure 2). These data strongly indicate that PP2A is important in preserving sIL-2R $\beta$ on the surface of Tregs.

$P P 2 A$ prevents $I L-2 R \beta$ shedding from the surface membrane of thymic $C D 4^{+}$Tregs. Alternative splicing and shedding are both classical mechanisms of producing soluble forms of membrane proteins. The gene $I l 2 \mathrm{rb}$ 

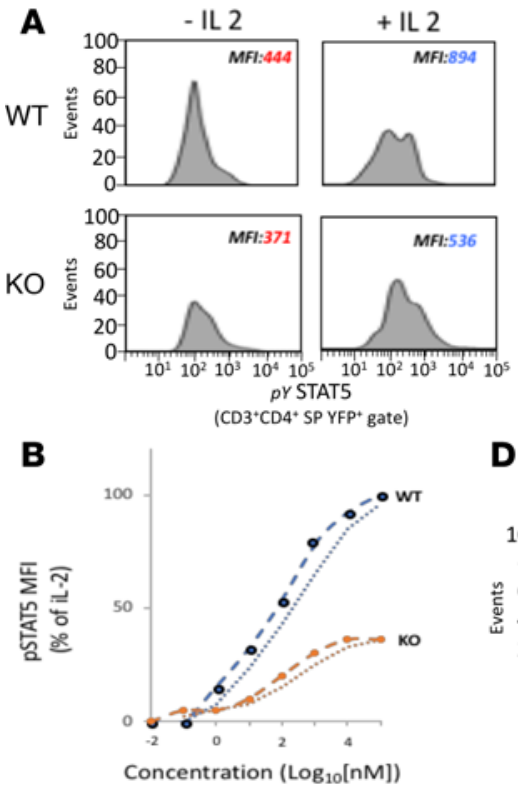

\begin{tabular}{c|ccc|} 
& \multicolumn{3}{|c|}{${\text { pSTAT5 } \text { EC }_{50}}$} \\
\hline & KO & WT & RATIO \\
& EC $_{50}$ & EC $_{50}$ & (KO/ \\
& $(\mathrm{nM})$ & $(\mathrm{nM})$ & WT) \\
\hline IL-2 & $\infty$ & 70 & WT \\
\hline
\end{tabular}

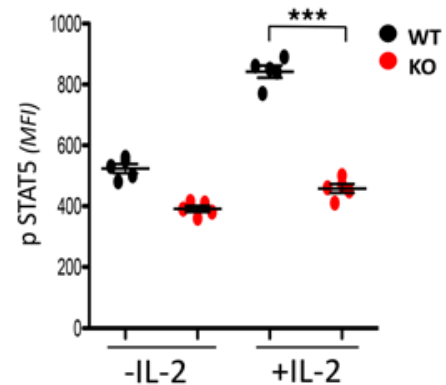

D
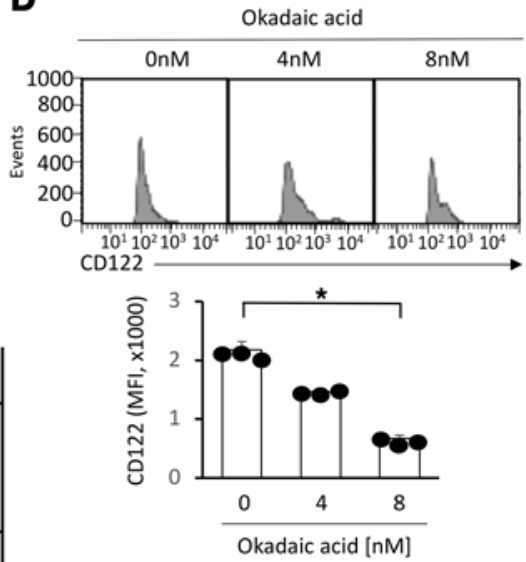

C
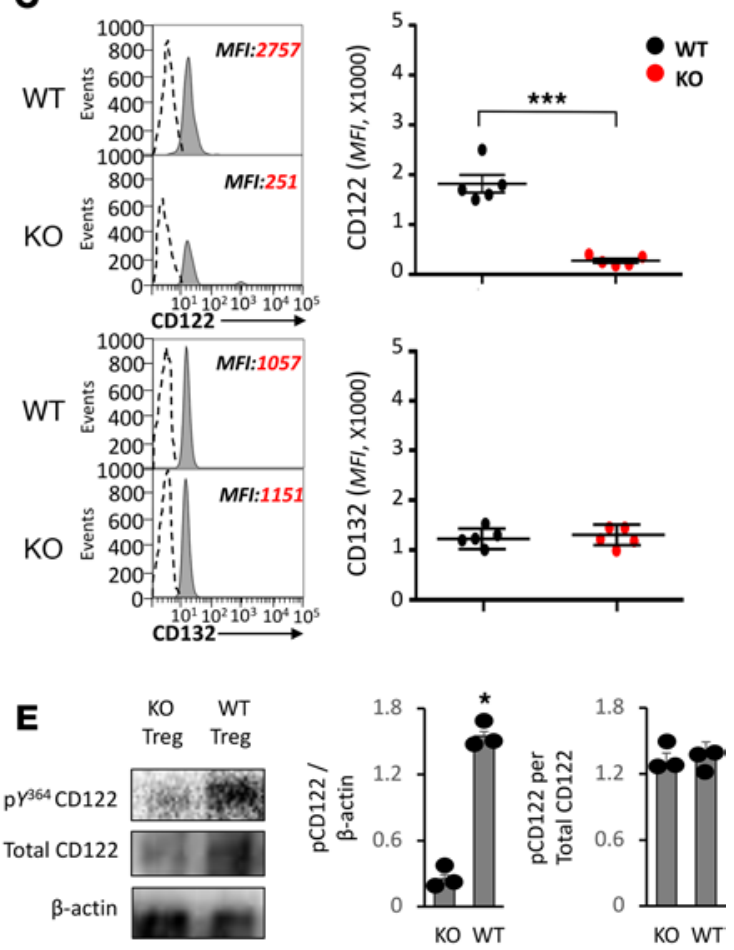

Figure 2. PP2A controls IL-2Rß expression and IL-2 signaling in thymic CD4+ Tregs. (A) Phosphorylated STAT5 (p-STAT5) following stimulation with IL-2 (20 mM, 20 minutes) in PP2A-sufficient (WT) and PP2A-deficient (KO) thymic CD4+ Tregs from individual mice ( $n=4-5 /$ group). Representative histograms and cumulative data (mean \pm SEM) from a representative of 3 experiments are shown. (B) Nonlinear regression analysis of dose-response curves (top) reflecting the MFI levels of p-STAT5 in WT and KO thymic CD4+ Tregs following stimulation with a dose range of IL-2 and a table for p-STAT5 EC ${ }_{50}$ values as calculated from these curves (bottom) from a representative of 3 biological replicates. (C) Expression of the IL-2R $\beta$ chain (CD122) and IL-2R $\gamma$ chain (CD132) in thymic CD4+ Tregs from WT and KO mice ( $n=5$ /group). Representative histograms and cumulative data (mean \pm SEM) from a representative of 3 experiments are shown. Dashed-line histograms represent negative control. (D) IL-2R $\beta$ expression in thymic CD4+ Tregs from WT mice ( $n=3$ ) following incubation with the PP2A inhibitor okadaic acid. Representative histograms and cumulative data (mean \pm SEM) from a representative of 3 experiments are shown. (E) Tyrosine phosphorylation ( $p-Y^{364}$ ) of CD122 and total levels of CD122 and $\beta$-actin in thymic CD4+ Tregs as detected by Western blot analysis of lysates that were pooled from WT and KO mice ( $n=3-4$ /group). Representative blots and cumulative data (mean \pm SEM) from 2 independent experiments are shown. ${ }^{*} P<0.05 ;{ }^{* * *} P<0.001$.

is composed of 9 exons, and the entire transmembrane domain (TM) is encoded by exon 7 (Figure 3E). Thus, alternative splicing of $I l 2 r b$ that excludes exon 7 would favor a soluble form of the IL-2R $\beta$ chain. To this end, primers 1 and 2, which encompass the expression of exon 7 alone, were designed to identify the TM domain of the IL-2R $\beta$. As shown in Figure 3E, both WT and KO thymic CD4 $4^{+}$Tregs expressed comparable levels of TM mRNA, a finding that ruled out alternative splicing of $I l 2 r b$ as a source of high sIL-2R $\beta$ chain level in the culture supernatants from the $\mathrm{KO} C D 4^{+}$Tregs.

To study the role of shedding in the formation of IL-2R $\beta$, KO thymic CD4+ Tregs were stimulated with IL-2 either alone or in the presence of the pan-sheddase inhibitor TAPI-2. Indeed, sheddase inhibition recovered both the surface expression (Figure 3F) and function (Figure 3G) of IL-2R $\beta$ on the surface membrane of PP2A-deficient $\mathrm{CD}^{+}$Tregs as assessed by the increased levels of p-STAT5. To confirm the involvement of PP2A in the shedding of IL-2R $\beta$, the response of WT thymic CD4 ${ }^{+}$Tregs to IL-2 was assessed in the presence of the PP2A inhibitor okadaic acid and the pan-sheddase inhibitor TAPI-2. As shown in Figure 3H, inhibition of PP2A led to decreased surface levels of the IL-2R $\beta$ chain, and the addition of IL-2 did not result in receptor internalization because the phosphorylation of STAT5 was not increased and was kept at baseline levels.

Interestingly, however, under these conditions of induced IL-2R $\beta$ deficiency, the highest concentrations of sheddase inhibitor could overcome the repressing effects of PP2A deficiency on the surface IL-2R $\beta$ chain expression and restore its responsiveness to IL-2 as indicated by the increase in STAT5 phosphorylation. Sheddase inhibition itself (e.g., wherein surface IL-2R $\beta$ was sufficient and not manipulated) did not 


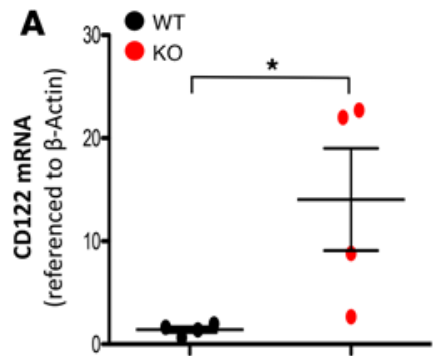

B

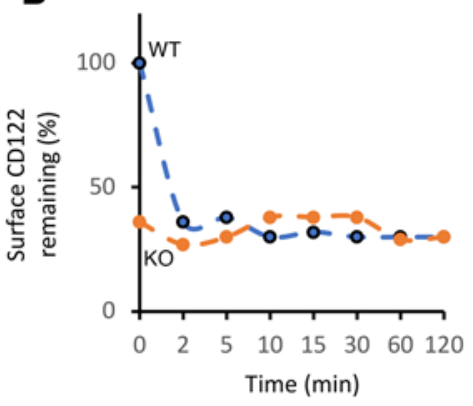

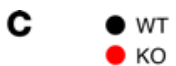

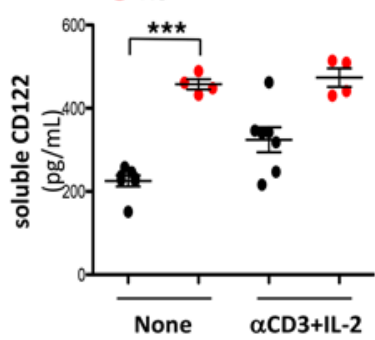

D $\quad$ WT

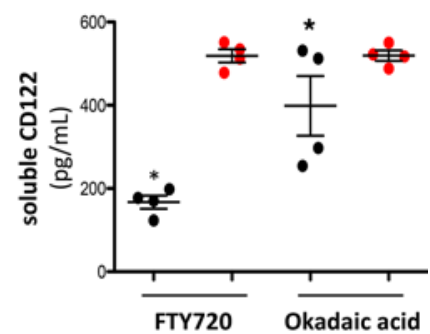

E

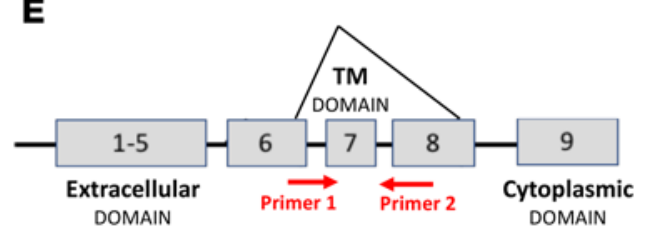

WT KO

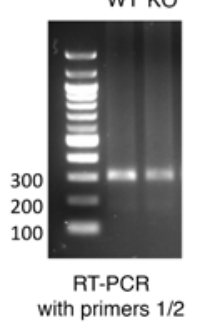

TM, IL-2R $\beta$

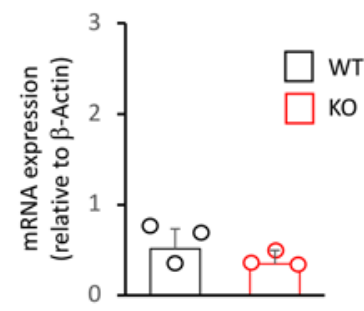

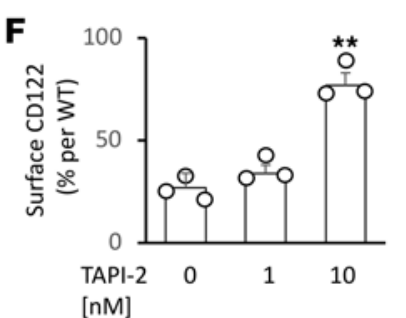
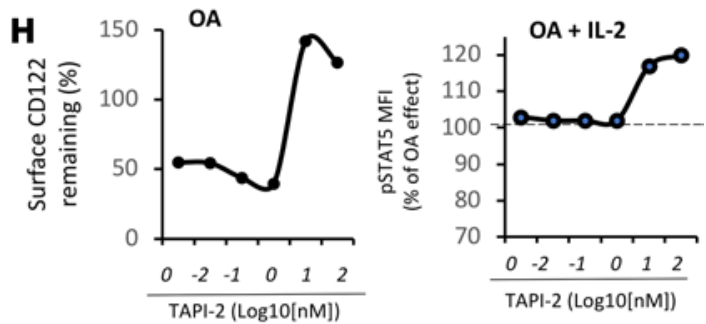

G

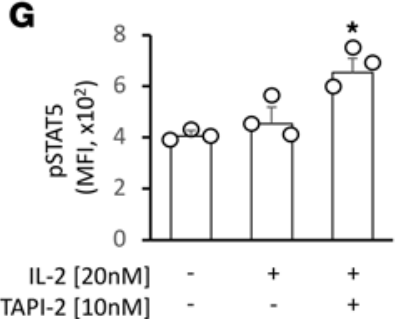

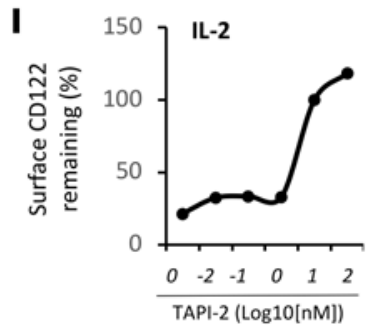

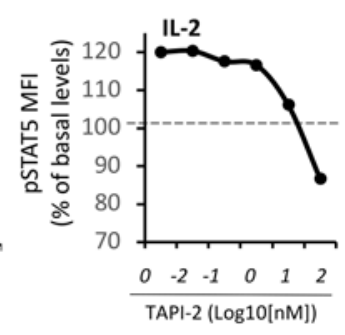

Figure 3. PP2A prevents IL-2R $\beta$ shedding and the formation of soluble IL-2R $\beta$ by thymic CD4+ Tregs. (A) IL-2R $\beta$ per $\beta$-actin mRNA expression (mean \pm SEM) in thymic CD4 ${ }^{+}$Tregs from WT and KO mice ( $n=4$ /group). (B) Baseline levels of surface IL-2R $\beta$ in WT (considered as $\left.100 \%\right)$ and KO (relative to WT) thymic $\mathrm{CD}^{+}$Tregs and time kinetics in surface IL-2R $\beta$ remaining after IL-2 stimulation (20 nM) as detected by flow cytometry. (C) Thymic CD4+ Tregs from KO and WT mice were cultured overnight in full medium alone or in $\alpha$-CD3-precoated plates in the presence of IL-2 or (D) in the presence of the PP2A inhibitor okadaic acid or the PP2A activator FTY720, and thereafter the levels of sIL-2R $\beta$ were determined in the supernatants using ELISA. Statistical analysis is relative to unstimulated thymic CD4+ Tregs from WT mice. (E) Schematic of primer design and alignment for mouse II2rb mRNA expression. Total RNA from thymic CD4 Tregs from KO and WT mice ( $n=3-4$ /group) was assessed for mRNA transcripts of the TM domain of IL-2R $\beta$ using quantitative real-time PCR. Cumulative data (mean \pm SEM) from 3 experiments are shown. (F) Surface IL-2R $\beta$ (relative to WT) and (C) p-STAT5 expression in thymic CD4+ Tregs from KO mice following stimulation with IL-2 (20 nM, 20 minutes) and the pan-sheddase inhibitor TAPI-2. (H) Surface IL-2R (relative to baseline) in thymic WT Tregs after PP2A inhibition with okadaic acid (OA) and in the presence of the pan-sheddase inhibitor TAPI-2 (left end) and p-STAT5 expression relative to OA effect (represented by the dashed line) in response to IL-2 (right end). (I) Surface IL-2R $\beta$ (left end) and p-STAT5 expression (right end) relative to baseline levels (represented by the dashed line) in thymic WT Tregs following IL-2 stimulation and pan-sheddase inhibition with TAPI-2. ${ }^{*} P<0.05 ;{ }^{* *} P<0.005 ;{ }^{* *} P<0.001$.

improve further IL-2 signaling, because both IL-2R $\beta$ internalization and STAT5 phosphorylation occurred and were maintained regardless of sheddase inhibition (Figure 3I). Yet, the highest concentrations of sheddase inhibition interfered with IL-2R $\beta$ internalization and function because the surface IL-2R $\beta$ expression increased, and STAT5 phosphorylation decreased, despite IL-2 stimulation.

$P P 2 A$ affects $I L-2$ signaling in peripheral $C D 4^{+}$Tregs by repressing the formation of soluble IL-2R. Spleen CD4 ${ }^{+}$ Tregs were studied to determine whether PP2A affects IL-2R $\beta$ function in the periphery. It can be seen in Figure $4 \mathrm{~A}$ that $\mathrm{KO} \mathrm{CD}^{+}$Tregs from the spleen responded to low concentrations of IL-2 similarly as WT $\mathrm{CD}^{+}$Tregs but lagged behind $(P<0.001)$ at higher concentrations of IL-2. As in the thymus, the baseline levels of sIL-2R $\beta$ chain in supernatants of $\mathrm{KO} \mathrm{CD} 4^{+}$Tregs from the spleen were significantly higher compared with those of WT CD4 ${ }^{+}$Tregs (Figure 4B). The presence of the pan-sheddase inhibitor TAPI-2 increased the expression of IL-2R $\beta$ on the surface membrane in a dose-dependent manner (Figure $4 \mathrm{C}$ ) and improved the response to IL-2, as indicated by the increased levels of p-STAT5 in the KO CD4+ Tregs (Figure 4D). The loss of surface IL-2R in the $\mathrm{KO}$ mice was due to PP2A deficiency in the CD4 ${ }^{+}$Tregs because incubation of WT CD4 ${ }^{+}$Tregs with okadaic acid also decreased surface IL-2R $\beta$ and interfered with IL-2 signaling (Figure 4E). Sheddase inhibition with TAPI-2 restored the okadaic acid-induced reduction of IL-2R $\beta$ and restored the receptor function in response to IL-2 (Figure 4E). 
A

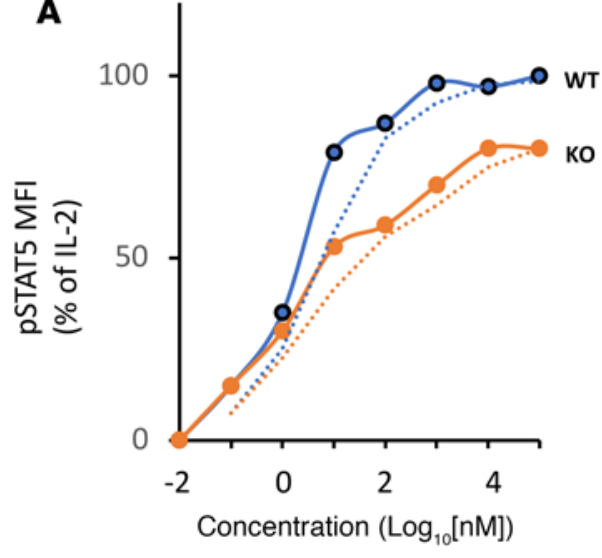

B

\begin{tabular}{|c|c|c|c|}
\hline & \multicolumn{3}{|c|}{ pSTAT5 EC 50} \\
\hline & $\begin{array}{l}\text { KO } \\
\mathrm{EC}_{50} \\
\text { (nM) }\end{array}$ & $\begin{array}{l}\text { WT } \\
\mathrm{EC}_{50} \\
\text { (nM) }\end{array}$ & $\begin{array}{c}\text { RATIO } \\
\text { (KO/ } \\
\text { WT) }\end{array}$ \\
\hline IL-2 & 30 & 10 & 3 \\
\hline
\end{tabular}
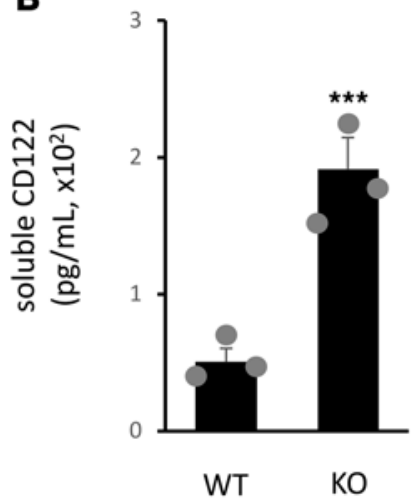

C

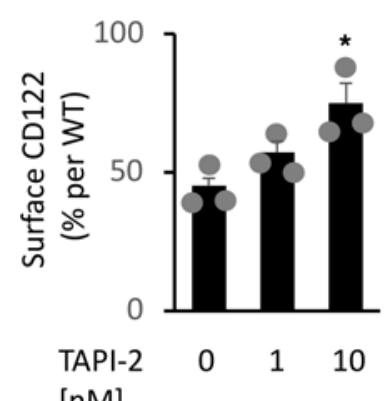

[nM]
D

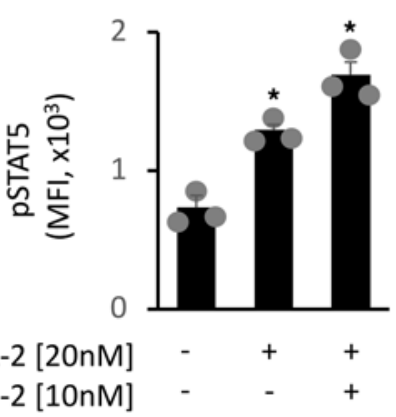

E

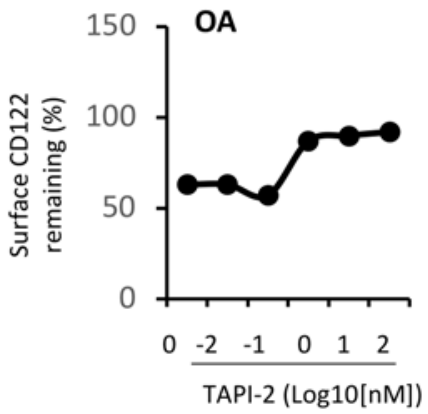

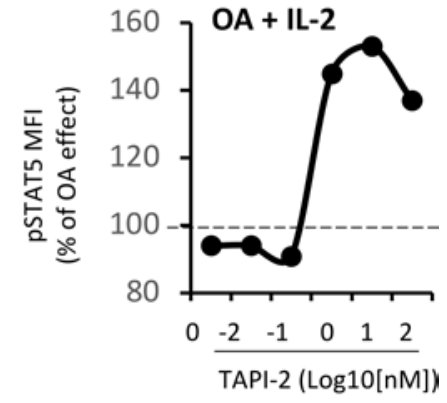

Figure 4. PP2A promotes IL-2 signaling in spleen-derived CD4+ ${ }^{+}$Tregs by repressing the formation of sIL-2Rß. (A) Dose-response curves (left end) reflecting the MFI levels of $\mathrm{p}-\mathrm{STAT5}$ in spleen-derived CD4+ Tregs from WT and KO mice following stimulation with a dose range of IL-2 and a table for $\mathrm{p}-\mathrm{STAT5}$ $\mathrm{EC}_{50}$ values (right end) from a representative of 3 biological replicates. (B) sIL-2R $\beta$ in the supernatants of spleen-derived CD4+ Tregs from WT and KO mice following overnight incubation in full medium. (C) Surface IL-2R $\beta$ (relative to WT) and (D) p-STAT5 expression in spleen-derived CD4+ Tregs from KO mice in the presence of the pan-sheddase inhibitor TAPI-2 alone (C) or in combination (D) with IL-2 stimulation (20 nM, 20 minutes). (E) Surface IL-2R $\beta$ (relative to baseline) in spleen-derived WT Tregs after PP2A inhibition with OA and in the presence of the pan-sheddase inhibitor TAPI-2 (left end) and p-STAT5 expression relative to $\mathrm{OA}$ effect in response to IL-2 (right end). ${ }^{*} P<0.05 ;{ }^{* *} P<0.001$.

Replenishment of IL-2R $\beta$ levels in PP2A-deficient CD4 $4^{+}$Tregs restores IL-2 signaling. To address directly whether increased shedding of IL-2R $\beta$ was linked to absence of PP2A, PP2A-deficient or -sufficient CD4 $4^{+}$Tregs were infected with 3 amounts (low, medium, and high) of mCherry-lentiviral mouse IL-2R $\beta$ (pCD122) or empty vector $(\mathrm{pEV})$. Twenty-four hours later the cells were exposed to IL-2 for 20 minutes, and the p-STAT5 levels were assessed by flow cytometry. As shown in Figure 5A, phosphorylation of STAT5 originated from 2 distinctive cell populations, low-mCherry expressing (shown as a green rectangle) and highmCherry expressing (shown as a red rectangle) $\mathrm{CD} 4^{+}$Tregs, depicting the native and the infected IL-2R $\beta$ chain, respectively. Figure 5B presents schematically STAT5 phosphorylation from the 2 sources of receptors, the native and the infected IL-2R $\beta$, following infection with either $\mathrm{pCD} 122$ or the empty vector ( $\mathrm{pEV}$ ). WT CD4 ${ }^{+}$Tregs infected with $\mathrm{pEV}$ signaled through their native IL-2R $\beta$ to yield STAT5 phosphorylation but $\mathrm{KO} \mathrm{CD} 4^{+}$Tregs did not. CD4 ${ }^{+}$Tregs from KO mice, though infected with pCD122 displayed increasing STAT5 phosphorylation, reaching the levels of WT Tregs when infected with the highest doses of pCD122. In addition, replenishment of CD122 increased the signaling capacity through the native CD122 (Figure 5B). Altogether, PP2A deficiency in peripheral CD4 ${ }^{+}$Tregs critically interferes with IL-2 signaling, and signaling can be resumed effectively by sheddase inhibition or forced expression of IL-2R $\beta$.

$A D A M 10$ is increased in thymic PP2A-deficient $C D 4^{+}$Tregs, and inhibition of ADAM10 reduces sIL-2R $\beta$ and restores the surface expression of $I L-2 R \beta$ and $I L-2$ signaling. Sheddases mediate the formation of sIL-2R $\beta$ in $\mathrm{CD}^{+}$Tregs that are deficient in PP2A. Proteins with ectodomain sheddase activity that are also TM are potential candidates for executing the cleavage of the extracellular domain of sIL-2R $\beta$ (25). We screened 
A
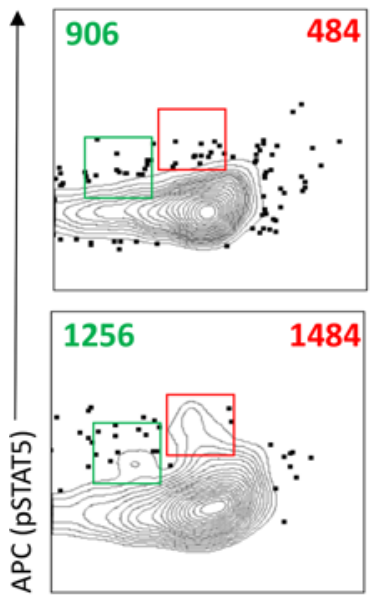

mCherry (IL-2R $\beta$ chain)
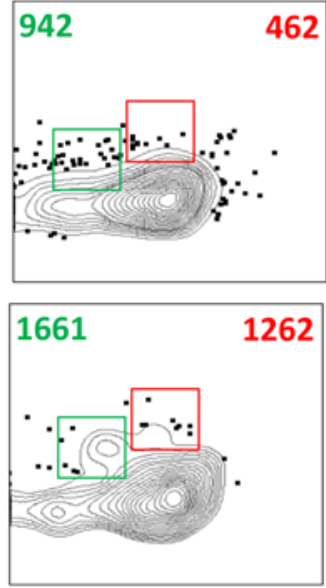
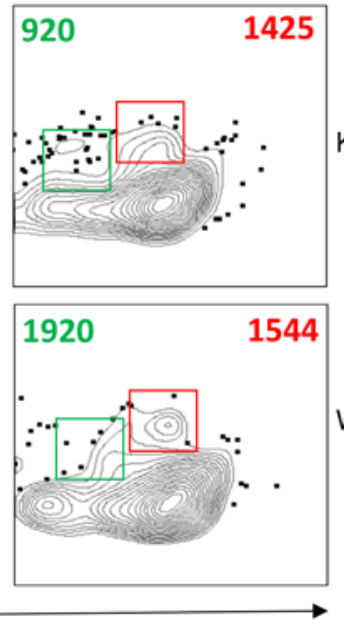

KO

B Vector: $\mathrm{pEV}$

Output: pSTAT5 via native CD122

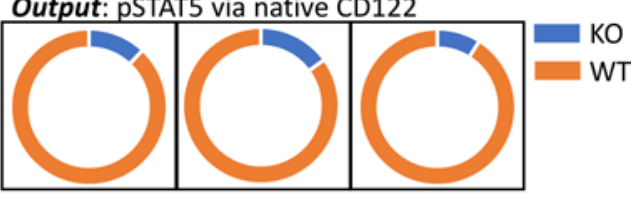

Vector: $\mathrm{pCD} 122$

Output: pSTAT5 via pCD122

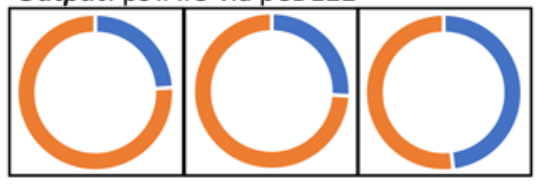

Vector: $\mathrm{pCD} 122$

Output: pSTAT5 via native CD122

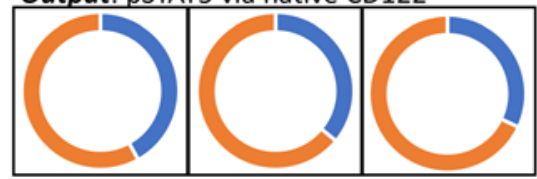

Low

High

\section{IL-2R $\beta$ chain}

or empty vector (MOI)

Figure 5. Replenishment of IL-2R $\beta$ levels in KO CD4+ Tregs restores IL-2 signaling. (A) Expression of $p-S T A T 5$ following infection with 3 amounts (low, medi-

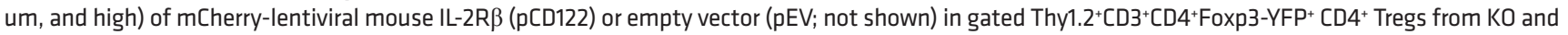
WT mice. Twenty-four hours later the cells were exposed to IL-2 (20 nM, 20 minutes) and p-STAT5 was assessed by flow cytometry. Low-mCherry expressing (shown as a green rectangle) and high-mCherry expressing (shown as a red rectangle) CD4+ Tregs reflect the p-STAT5 via the native and the infected IL-2R $\beta$ chain, respectively. (B) Schematic presentation of p-STAT5 in KO Tregs either via the native IL-2R $\beta$ following infection with pEV (upper row) or via the infected (middle row) and native IL-2R $\beta$ (lower row) following infection with pCD122. APC, allophycocyanin; MOI, mount of infection.

sheddases from the ADAM family because they are TM molecules, and we found 5 out of 6 present in thymic $\mathrm{CD} 4^{+}$Tregs and noted that their mRNA expression was increased in the absence of PP2A (Figure 6A). ADAM10 expression was exceptionally high (20-fold). Incubation of thymic WT CD4 ${ }^{+}$Tregs with the PP2A activator FTY720 did not affect the mRNA expression of ADAM10; however, inhibition of PP2A with okadaic acid led to a significant increase in ADAM10 expression (Figure 6B). Specific inhibition of ADAM10 (GI254023X) resulted in reduction of sIL-2R $\beta$ in the supernatants from thymic PP2A-deficient $\mathrm{CD} 4^{+}$Tregs in a dose-dependent manner (Figure $6 \mathrm{C}$ ) and recovery of the expression of IL-2R $\beta$ on the cell surface membrane (Figure 6D, broken-line histogram) and enabled its internalization (Figure 6D, light-gray histogram) following stimulation with IL-2 (20 nM for 20 minutes). To confirm that PP2A deficiency resulted in increased ADAM10 activity, thymic $\mathrm{CD} 4^{+}$Tregs sufficient in PP2A were incubated in the presence of okadaic acid, and this led to increased levels of sIL-2R $\beta$ in the supernatants. However, the effect of okadaic acid was abolished when the ADAM10 inhibitor was added to the cultures and the level of sIL-2R $\beta$ was reduced (Figure $6 \mathrm{E}$ ). The potential function of the recovered IL-2R $\beta$ on the surface of thymic PP2A-deficient $\mathrm{CD} 4^{+}$Tregs by ADAM10 inhibitor was tested by stimulating the cells with IL-2, and indeed, the phosphorylation of STAT5 increased significantly $(P<0.005)$ in the presence of the ADAM10 inhibitor (Figure 6F). ADAM10 inhibition in PP2A-sufficient thymic CD4 $4^{+}$Tregs did not further increase the IL-2-induced phosphorylation of STAT5 beyond that observed in the PP2A-deficient CD4+ Tregs.

\section{Discussion}

In this study we present evidence that PP2A is required to enable Foxp3 expression and to maintain higher numbers of Tregs in the thymus by preventing the selective loss of surface IL-2R $\beta$ and preserving the IL-2R signaling potency in Tregs. Lentiviral overexpression of mouse IL-2R $\beta$ in PP2A-deficient Tregs can overcome the decreased expression of IL-2R and resume IL-2 signaling. The loss of IL-2R $\beta$ in Tregs is due to increased sheddase activity, mostly that of ADAM10, and both pan-sheddase inhibition and selective ADAM10 inhibition can restore IL-2R $\beta$ expression and signaling. The association of PP2A with sheddase activity and IL-2R expression in Tregs is novel to our knowledge and is described here for the first time. 
A

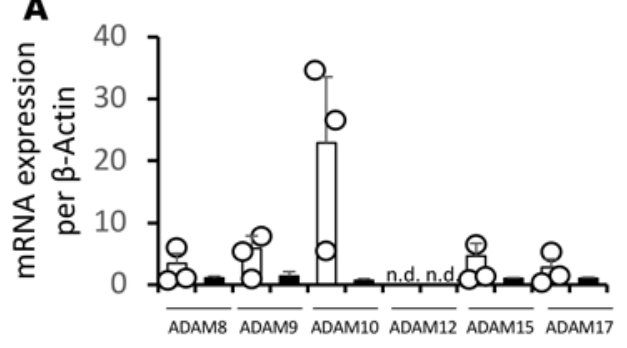

B

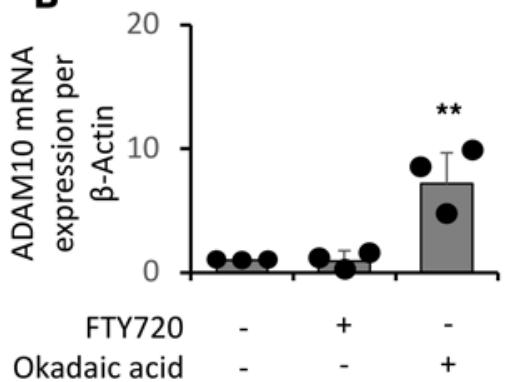

Okadaic acid - $\quad$ -
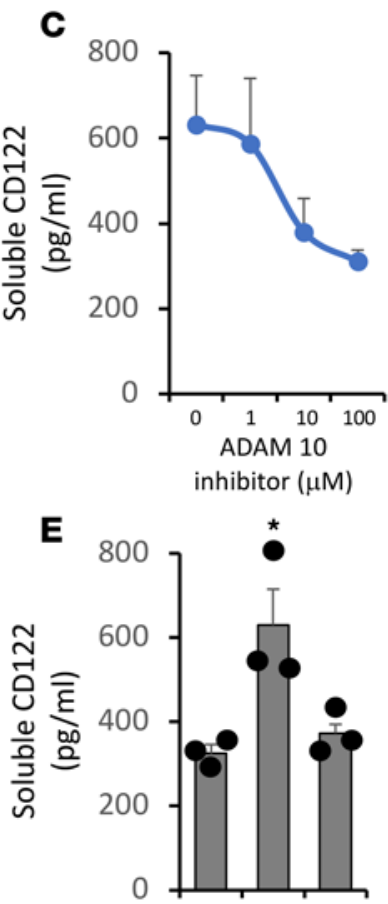

Okadaic acid [4nM] - + +
D

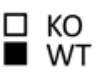

\section{ADAM10} IL-2 inhibitor
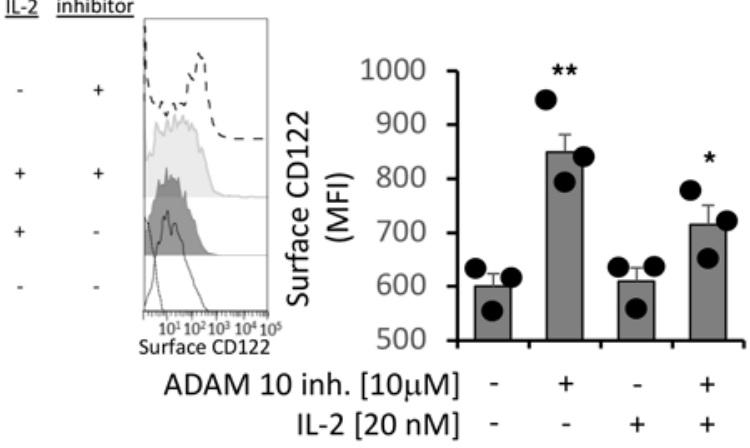

$\mathbf{F}$

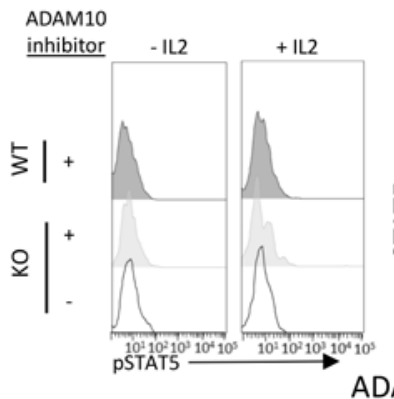

ADAM 10 inh.

[10 $\mu \mathrm{M}]$

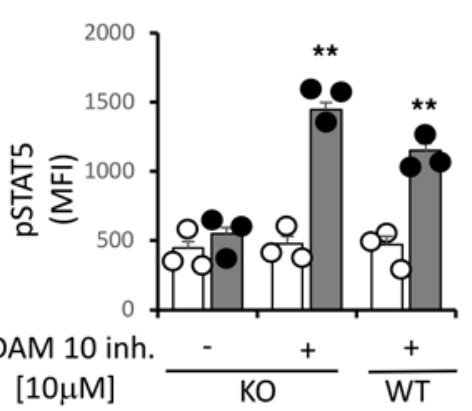

Figure 6. ADAM10 selectively cleaves surface IL-2R $\beta$ in CD4 ${ }^{+}$Tregs, and PP2A inhibits ADAM10 and restores IL-2 signaling. (A) Thymic CD4 ${ }^{+}$Tregs from KO and WT mice $(n=3)$ were screened for the mRNA expression of members in the ADAM family, and cumulative data (mean \pm SEM) from 3 experiments are shown. (B) The mRNA expression of ADAM10 relative to $\beta$-actin in thymic CD4+ Tregs from WT mice $(n=3)$ that were incubated with the PP2A activator FTY720 $(5 \mu \mathrm{M})$ or with the PP2A inhibitor OA (4 nM) for 4 hours. (C) The levels of soluble IL-2R $\beta$ in the supernatants of thymic CD4 ${ }^{+}$Tregs from KO mice ( $n$ $=3$ ) in the presence of the selective inhibitor ADAM10 (CI254023X) in different doses. (D) Representative flow cytometry histograms depicting the surface IL-2R $\beta$ expression in thymic CD4+ Tregs from KO mice $(n=3)$ at baseline (accompanied by a dotted-line histogram representing the negative control) or in response to ADAM10 inhibitor (CI254023X; $10 \mu \mathrm{M}$; broken-line histogram), IL-2 (20 nM; dark-gray histogram), or their combination (light-gray histogram). Cumulative data (mean \pm SEM) from 3 experiments are shown. (E) Soluble IL-2R $\beta$ in the supernatants of thymic CD $4^{+}$Tregs from WT mice $(n=3)$ that were incubated with PP2A inhibitor OA (4 nM), ADAM10 inhibitor (GI254023X; $10 \mu \mathrm{M})$, or their combination. Cumulative data (mean \pm SEM) from 3 experiments are shown. (F) Expression of p-STAT5 following ADAM10 inhibition (GI254023X; $10 \mu \mathrm{M})$ and IL-2 stimulation (20 nM) in thymic Tregs from WT ( $n=3$; darkgray histogram) and $\mathrm{KO}(n=3$; light-gray histogram) mice. Cumulative data (mean \pm SEM) from 3 experiments are shown.

We found that PP2A is involved in the development of Tregs in the thymus, as indicated by the decreased frequency of $\mathrm{CD} 4^{+}$Tregs along with reduced expression of Foxp 3 in the thymus from mice in which only Tregs lacked PP2A. Thymocytes express PP2A at very early stages of their development (starting from double-negative stage 2a; A. Sharabi, unpublished observations), and premature CD4 ${ }^{+}$ Tregs express PP2A before the appearance of Foxp3. TCR and IL-2R $\alpha$ are required for inducing the expression of Foxp3 in the developing $\mathrm{CD}^{+}$Tregs in the thymus (21), and both are maintained when deletion of loxP-flanked $P P 2 A$ alleles $\left(P P 2 A^{\mathrm{fl} / \mathrm{fl}}\right)$ is mediated by Cre recombinase driven by the endogenous Foxp3 locus $\left(F o x p 3^{\text {Cre }}\right)$. Thus, the aberrant development of CD4 ${ }^{+}$Tregs when PP2A is deficient originates from stages later than those stages involving expression of TCR and IL-2R $\alpha$.

STAT5 signaling is a key player for the differentiation of Treg precursors that have permissive TCR 
signaling (21). STAT5 is involved in the induction and maintenance of Foxp3 expression by binding to the Foxp3 promoter and the intronic Foxp3 regulatory element CNS2 (26). Although PP2A-deficient CD4 ${ }^{+}$ Tregs required TCR and IL-2R $\alpha$ to differentiate to Foxp3-expressing CD4 ${ }^{+}$Tregs, the levels of p-STAT5 were reduced and did not increase in response to IL-2. CD4 ${ }^{+}$Tregs that are deficient in STAT5 exhibit a severe impairment of suppressor function (27). Dose-response curves indicated that IL-2R potency was severely compromised in thymic PP2A-deficient CD4 ${ }^{+}$Tregs. Deficiency of PP2A in CD4 ${ }^{+}$Tregs resulted in upregulation of the IL-2R $\alpha$ chain expression without affecting the expression and function of the IL-2R $\gamma$ chain; however, it led to a substantial diminished expression of the IL-2R $\beta$ chain that mediates the phosphorylation of STAT5, thus interfering with the capacity of the cells to induce Foxp3. Because IL-2R $\beta$ is also shared with the IL-15 receptor (28), the diminished expression of IL-2R $\beta$ could also interfere with IL-15 receptor signaling, which contributes in part to the induction of Foxp3 in the thymus (29).

The significance of the $\beta$ chain of the IL-2R has been reported to be mainly in the thymus and less in the periphery. This finding was observed in $I l 2 \mathrm{rb}^{-/-}$mice that develop lethal autoimmune disease; however, this phenotype could be prevented with the expression of a transgene encoding IL-2R $\beta$ exclusively in thymocytes $(2,6)$. Similarly, deletion of IL-2R $\beta$ only in Tregs (Foxp3 $3^{\text {re }} I l 2 r b^{\mathrm{f} / \mathrm{fl}}$ mice) resulted in the development of systemic, fatal autoimmune inflammatory lesions and lymphoproliferation (27), and both the frequency of CD4 ${ }^{+}$ Tregs and the expression of Foxp3 per cell were lower, with a loss of suppressive function in vivo.

Low levels of IL-2R $\beta$ on the surface of thymic PP2A-deficient CD4 $4^{+}$Tregs were associated with increased levels of sIL-2R $\beta$. Membrane metalloproteinases have been linked to the production of sIL-2R $\alpha$ (30) and preferential alternative splicing for the soluble form of the $I l 2 \mathrm{rg}$ gene (31). There are no reports to our knowledge on the formation of sIL-2R $\beta$. Here we found that the production of sIL-2R $\beta$ by PP2A-deficient CD4 ${ }^{+}$Tregs did not involve the alternative splicing of the $I l 2 r b$ gene, but rather it was the result of increased sheddase activity.

ADAMs are TM and secreted proteins that contain metalloproteinase domains and are involved in "ectodomain shedding" of diverse growth factors, cytokines, receptors, and adhesion molecules (25). In humans only 13 of the 21 genes in the family encode functional proteases, and in mice 6 of them are TM and considered full-time sheddases. Screening for ADAMs revealed that 5 of the 6 ADAMs could be detected at higher levels in PP2A-deficient CD4 ${ }^{+}$Tregs, and among them, ADAM10 was expressed the highest. PP2A was found to control ADAM10 activity because PP2A inhibition (using okadaic acid) and not PP2A promotion (using FTY720) led to the upregulation of ADAM10, and ADAM10 mediated the production of sIL-2R $\beta$ in the PP2A-deficient CD4 ${ }^{+}$Tregs. More important, specific inhibition of ADAM10 was capable of inhibiting the formation of sIL-2R $\beta$ and resuming the phosphorylation of STAT5. As low-dose IL-2 is being introduced for the treatment of autoimmune disease (32), inhibition of ADAM10 may represent a therapeutic adjuvant.

Our findings highlight a central role for PP2A in IL-2R signaling in thymic CD4 ${ }^{+}$Tregs that is important for their development and maintenance. PP2A prevents the ectodomain cleavage of the IL-2R $\beta$ chain by the TM sheddase ADAM10 and preserves IL-2R $\beta$ on the cell surface. In the presence of PP2A the IL-2R's potency is maximized in the $\mathrm{CD}^{+}$Tregs, and the cells can effectively respond to low concentrations of IL-2 and outcompete effector T cells when IL-2 is limited.

\section{Methods}

Mice. As previously described (17), the dominant $\alpha$-isoform (Ppp2r1a) of the scaffold PP2AA subunit was deleted in a Treg-specific manner by crossing Foxp $3^{Y F P-c r e}$ mice with Ppp2r1a $a^{\mathrm{f} / \mathrm{fl}}$ mice to generate Foxp $3^{Y F P-c r e}$ $P p p 2 r 1 a^{\mathrm{f} / \mathrm{fl}}$ (termed here $\mathrm{KO}$ mice) and Foxp3 $3^{Y F P-c r e} P p p 2 r 1 a^{+/+}$(termed here WT mice). All mice were bred and housed in a specific pathogen-free environment in a barrier facility in accordance with the Beth Israel Deaconess Medical Center Institutional Animal Care and Use Committee (IACUC).

Treg isolation and stimulation. Thymus and spleen Tregs were isolated by sorting (more than $95 \%$ purity) on an FACSAria II (Becton Dickinson) as Thy $1.2^{+} \mathrm{CD}^{+}{ }^{+} \mathrm{CD}^{+} \mathrm{CD} 8^{-} \mathrm{CD} 25^{+} \mathrm{Foxp}^{-} \mathrm{YFP}^{+} \mathrm{T}$ cells. Cells were stimulated in full RPMI 1640 with plate-bound mouse anti-CD3 $(1 \mu \mathrm{g} / \mathrm{ml}$, clone $145-2 \mathrm{C} 11$; BioLegend $)$ and mouse IL-2 (20 nM; R\&D Systems). In some experiments okadaic acid, FTY720, ADAM10 inhibitor GI254023X (MilliporeSigma), and TAPI-2 (Cayman Chemical) were used.

Western blot analysis. We applied established protocols (17) and used the following antibodies for Western blotting: anti-CD122 antibody, $\mathrm{pY}{ }^{364} \mathrm{CD} 122$, and Foxp3 from Invitrogen; antiactin from MilliporeSig$\mathrm{ma}$; and goat anti-rabbit IgG coupled with HRP from Jackson Immunoresearch.

Flow cytometry. Lymphocytes were stained for dead cells (Zombie Aqua/UV Fixable Viability Kit; BioLegend) and then were stained at $4^{\circ} \mathrm{C}$ in PBS containing $2 \%$ FBS and $0.5 \%$ EDTA after Fc $\gamma$ RII/III block- 
ing. The following fluorescent anti-mouse antibodies were administrated according to the manufacturer's instructions: Thy1.2 (clone 30H-12), CD4 (clone GK1.5), CD8 (clone 53-6.7), TCR $\beta$ (clone H57-597), CD3 (clone 145-2C11), CD25 (clone BC96), CD122 (clone TU27), CD132 (clone TUGm2). All antibodies were purchased from BioLegend. For $\mathrm{pY}^{694}$ STAT5 (clone C71E5; Cell Signaling Technology), cells were permeabilized using the PerFix EXPOSE Kit (Beckman Coulter). Data were acquired on an LSR II SORP digital cell analyzer (BD Biosciences) and analyzed using FlowJo version 10.0.8.

IL-2R $\beta$ chain internalization. IL-2 $(20 \mathrm{nM})$ was incubated with either $\mathrm{KO}$ or WT thymic Tregs for 0, 2, 5, $10,15,30,60$, and 120 minutes. Cells were immediately transferred to ice to prevent further receptor internalization and washed twice with ice-cold PBSA buffer (0.1\% BSA in PBS) and stained with 1:50 dilutions of CD122 in PBSA buffer for 30 minutes on ice. After 2 more washes in ice-cold PBSA buffer, cells were fixed for 10 minutes at room temperature with $1.5 \%$ paraformaldehyde in PBSA, washed, and resuspended in PBSA buffer. MFI values of CD122 at each time point were compared with the MFI value of WT thymic Tregs at time point 0 (considered as 100\%).

$R N A$ isolation and real-time PCR. RNA was isolated using RNeasy Mini Kit (Qiagen), and cDNA was produced using Maxima H Minus First Strand cDNA Synthesis Kit (Thermo Fisher Scientific) according to the manufacturers' protocols. Real-time PCR was performed using a LightCycler 480 SYBR Green I Master kit (Roche).

Lentivector construction and transduction. For mouse IL-2R $\beta$ lentivector construction, the full coding sequences of mouse IL-2R $\beta$ were generated from pScalps_puro_mIL-2R $\beta$ (plasmid 59916, Addgene) through digestion using XbaI and NotI restriction enzymes. Subsequently, the sequences were subcloned into XbaIand NotI-digested pLVX-EF1 $\alpha$-IRES-mCherry-lentiviral vector (catalog 631987, Clontech Laboratories). For the production of pLVX-EF1 $\alpha$-IRES-mCherry (empty vector) or pLVX-EF1 $\alpha$-IRES-mCherry-mIL-2R $\beta$ lentivirus, the lentivector, packaging plasmid psPAX2, and envelope plasmid pMD2.G were cotransfected at a ratio of 4:3:1 into HEK293 T cells using Lipofectamine 2000 (Invitrogene). At 48 hours after transfection, lentiviral particles in the supernatant were collected, filtered through a $0.45-\mu \mathrm{m}$ filter, and concentrated with Lenti-X Concentrator (catalog 631231, Clontech Laboratories). For lentiviral transduction, freshly concentrated lentiviral particles were spun-inoculated into naive $\mathrm{CD} 4^{+} \mathrm{T}$ cells supplemented with $8 \mu \mathrm{g} / \mathrm{ml}$ of polybrene (MilliporeSigma) at $805 \mathrm{~g}, 32^{\circ} \mathrm{C}$, for 90 minutes. Then the cells were stimulated with IL-2. Downstream flow cytometry analysis of the infected cells was performed by gating on $\mathrm{mCherry}^{+} \mathrm{FOXP} 3-\mathrm{YFP}^{+} \mathrm{T}$ cells.

Detection of soluble mouse IL-2R (CD122). Levels of soluble CD122 in supernatants from Treg cultures were determined using the Mouse IL-2sR $\beta / C D 122$ (Soluble Interleukin-2 Receptor) CLIA Kit (MyBioSource) according to the manufacturer's protocol (detection range $15.625-1,000 \mathrm{pg} / \mathrm{ml}$; sensitivity min: $9.375 \mathrm{pg} / \mathrm{ml}$; $\max : 1,000 \mathrm{pg} / \mathrm{ml})$.

Statistics. All results are shown as the mean \pm SEM. A 2-tailed, unpaired Student's $t$ test was used when only 2 groups were compared for statistical differences. For a comparison of more than 2 groups, the 1-way ANOVA was applied. $P$ values of less than 0.05 were considered significant.

Study approval. All animal procedures were approved by the IACUC of Beth Israel Deaconess Medical Center, Harvard Medical School.

\section{Author contributions}

AS and GCT designed experiments, analyzed data, and wrote the manuscript. AS, IRK, and WP performed the experiments. VRM contributed to experiment design, analyzed data, provided expertise, and reviewed the manuscript. IRK, HL, WP, EM, and MGT provided expertise and reviewed the manuscript.

\section{Acknowledgments}

This work was supported by NIH grants R01 AI068787 and AI136924 to GCT and AR068974 to VRM.

Address correspondence to: George C. Tsokos, 3 Blackfan Cir., Center for Life Sciences — 937, Boston, Massachusetts 02215, USA. Phone: 617.735.4160; Email: gtsokos@bidmc.harvard.edu. Or to: Amir Sharabi, Rabin Medical Center, Jabotinsky St., Petah-Tikva, Israel 4941492. Phone: 972.54.766.6006; Email: amirshar3@clalit.org.il or amirsharabi@post.tau.ac.i1.

1. Furtado GC, Curotto de Lafaille MA, Kutchukhidze N, Lafaille JJ. Interleukin 2 signaling is required for CD4(+) regulatory T cell function. J Exp Med. 2002;196(6):851-857. 
2. Malek TR, Yu A, Vincek V, Scibelli P, Kong L. CD4 regulatory T cells prevent lethal autoimmunity in IL-2R $\beta$-deficient mice. Implications for the nonredundant function of IL-2. Immunity. 2002;17(2):167-178.

3. Fontenot JD, Rasmussen JP, Gavin MA, Rudensky AY. A function for interleukin 2 in Foxp3-expressing regulatory T cells. Nat Immunol. 2005;6(11):1142-1151.

4. Burchill MA, Yang J, Vogtenhuber C, Blazar BR, Farrar MA. IL-2 receptor $\beta$-dependent STAT5 activation is required for the development of Foxp3 ${ }^{+}$regulatory T cells. J Immunol. 2007;178(1):280-290.

5. Yao Z, et al. Nonredundant roles for Stat5a/b in directly regulating Foxp3. Blood. 2007;109(10):4368-4375.

6. Malek TR, Bayer AL. Tolerance, not immunity, crucially depends on IL-2. Nat Rev Immunol. 2004;4(9):665-674.

7. Setoguchi R, Hori S, Takahashi T, Sakaguchi S. Homeostatic maintenance of natural Foxp3(+) CD25(+) CD4(+) regulatory T cells by interleukin (IL)-2 and induction of autoimmune disease by IL-2 neutralization. J Exp Med. 2005;201(5):723-735.

8. Hatakeyama M, et al. Interleukin-2 receptor beta chain gene: generation of three receptor forms by cloned human alpha and beta chain cDNA's. Science. 1989;244(4904):551-556.

9. Takeshita T, et al. Cloning of the $\gamma$ chain of the human IL-2 receptor. Science. 1992;257(5068):379-382.

10. Stauber DJ, Debler EW, Horton PA, Smith KA, Wilson IA. Crystal structure of the IL-2 signaling complex: paradigm for a heterotrimeric cytokine receptor. Proc Natl Acad Sci U S A. 2006;103(8):2788-2793.

11. Wang X, Rickert M, Garcia KC. Structure of the quaternary complex of interleukin-2 with its alpha, beta, and gammac receptors. Science. 2005;310(5751):1159-1163.

12. Spolski R, Li P, Leonard WJ. Biology and regulation of IL-2: from molecular mechanisms to human therapy. Nat Rev Immunol. 2018;18(10):648-659.

13. Yu A, et al. Selective IL-2 responsiveness of regulatory T cells through multiple intrinsic mechanisms supports the use of lowdose IL-2 therapy in type 1 diabetes. Diabetes. 2015;64(6):2172-2183.

14. Xu Y, et al. Structure of the protein phosphatase 2A holoenzyme. Cell. 2006;127(6):1239-1251.

15. Shi Y. Serine/threonine phosphatases: mechanism through structure. Cell. 2009;139(3):468-484.

16. Sharabi A, Kasper IR, Tsokos GC. The serine/threonine protein phosphatase 2A controls autoimmunity. Clin Immunol. 2018;186:38-42.

17. Apostolidis SA, et al. Phosphatase PP2A is requisite for the function of regulatory T cells. Nat Immunol. 2016;17(5):556-564.

18. Fontenot JD, Rasmussen JP, Williams LM, Dooley JL, Farr AG, Rudensky AY. Regulatory T cell lineage specification by the forkhead transcription factor foxp3. Immunity. 2005;22(3):329-341.

19. Apostolou I, Sarukhan A, Klein L, von Boehmer H. Origin of regulatory T cells with known specificity for antigen. Nat Immunol. 2002;3(8):756-763.

20. Kishimoto H, Sprent J. Negative selection in the thymus includes semimature T cells. J Exp Med. 1997;185(2):263-271.

21. Lio CW, Hsieh CS. A two-step process for thymic regulatory T cell development. Immunity. 2008;28(1):100-111.

22. Fontenot JD, Dooley JL, Farr AG, Rudensky AY. Developmental regulation of Foxp3 expression during ontogeny. J Exp Med. 2005;202(7):901-906

23. Malek TR. The biology of interleukin-2. Annu Rev Immunol. 2008;26:453-479.

24. Yu A, Olosz F, Choi CY, Malek TR. Efficient internalization of IL-2 depends on the distal portion of the cytoplasmic tail of the IL-2R common gamma-chain and a lymphoid cell environment. J Immunol. 2000;165(5):2556-2562.

25. Weber S, Saftig P. Ectodomain shedding and ADAMs in development. Development. 2012;139(20):3693-3709.

26. Feng Y, Arvey A, Chinen T, van der Veeken J, Gasteiger G, Rudensky AY. Control of the inheritance of regulatory T cell identity by a cis element in the Foxp3 locus. Cell. 2014;158(4):749-763.

27. Chinen T, et al. An essential role for the IL-2 receptor in T(reg) cell function. Nat Immunol. 2016;17(11):1322-1333.

28. Waldmann TA. The multi-subunit interleukin-2 receptor. Annu Rev Biochem. 1989;58:875-911.

29. Vang KB, Yang J, Mahmud SA, Burchill MA, Vegoe AL, Farrar MA. IL-2, -7, and -15, but not thymic stromal lymphopoeitin, redundantly govern $\mathrm{CD}^{+}{ }^{+} \mathrm{Foxp}^{+}{ }^{+}$regulatory T cell development. J Immunol. 2008;181(5):3285-3290.

30. Levine SJ. Molecular mechanisms of soluble cytokine receptor generation. J Biol Chem. 2008;283(21):14177-14181

31. Hong $C$, et al. Activated T cells secrete an alternatively spliced form of common $\gamma$-chain that inhibits cytokine signaling and exacerbates inflammation. Immunity. 2014;40(6):910-923.

32. Sharabi A, Tsokos MG, Ding Y, Malek TR, Klatzmann D, Tsokos GC. Regulatory T cells in the treatment of disease. Nat Rev Drug Discov. 2018;17:823-844. 